\title{
O capital humano nos municípios paranaenses: uma análise com regressões quantílicas
}

\author{
Kassya Christina Keppe \\ Luciano Nakabashi $^{* *}$
}

\begin{abstract}
RESUMO - O presente trabalho trata-se do capital humano como um dos fatores determinantes para explicar a diferença no nível e crescimento do PIB por trabalhador nos municípios paranaenses. Muitos estudos mostraram que realmente existe uma relação positiva entre o capital humano e o crescimento da renda. Porém, o presente estudo mostra o efeito do capital humano nos diferentes quantis de renda, no período de 1980 a 2000. A análise empírica será realizada através de uma comparação entre os métodos de Regressões Quantilicas (LAD) e Mínimos Quadrados Ordinários (MQO).
\end{abstract}

Palavras-chave: Capital humano. Municípios paranaenses. Regressões quantílicas.

\section{INTRODUÇÃO}

O estado do Paraná apresenta grande disparidade na renda dos seus diferentes municípios. Muitos fatores podem explicar o crescimento da renda e, portanto, essa disparidade de renda entre os municípios, sendo que o capital humano é um dos possíveis candidatos. Isso ocorre porque a informação, o conhecimento e o grau de habilidade dos trabalhadores são fatores muito importantes no processo de produção, inovação e difusão de tecnologia.

Visando mensurar o impacto do capital humano no crescimento da renda. Mankiw, Romer e Weil ${ }^{1}$ (1992), incluíram o capital humano no modelo de crescimento endógeno de Solow (1956). Desde então, muitos estudos empíricos comprovaram esta relação.

O objetivo do presente estudo é apresentar os elementos que podem explicar a grande diferença de renda existente entre os municípios paranaenses para os anos de 1980, 1991 e 2000, utilizando o Método de Regressões Quantílicas, pois este permite analisar o impacto do capital humano em cada quantil da renda.

\footnotetext{
*Economista pela Universidade Federal do Paraná (UFPR). Endereço eletrônico: kckeppe@hotmail.com.

** Doutor em economia pelo Centro de Desenvolvimento e Planejamento Regional da Universidade Federal de Minas Gerais (CEDEPLAR/UFMG), professor do Departamento de Economia da Universidade Federal do Paraná (UFPR) e coordenador do Boletim Economia \& Tecnologia. Endereço eletrônico: Luciano.nakabashi@ufpr.br.

${ }^{1}$ MRW doravante.
} 
Além desta introdução, o estudo apresenta, na segunda seção a metodologia e a base de dados utilizados na análise empírica. Na terceira seção, fazemos uma análise dos resultados das regressões estimadas, sendo esta seguida por uma breve conclusão.

\section{METODOLOGIA E DADOS}

\subsection{O MÉTODO DE REGRESSÕES QUANTÍLICAS}

O método de Regressões Quantílicas fornece uma estrutura linear conveniente para examinar como os quantis de uma variável dependente muda em resposta a um conjunto de variáveis independentes. Dito de outro modo, a regressão quantílica permite a estimativa linear de funções quantílicas condicional.

Diferentemente do método de Mínimos Quadrados Ordinários ${ }^{2}$, que minimiza a soma dos quadrados dos resíduos, o método de Regressões Quantílicas, desenvolvido por Koenker e Basset (1978), minimiza a soma dos desvios absolutos, sendo, por esse motivo, também conhecido com estimador Least Absolute Deviations ${ }^{3}$.

Supondo a seguinte equação:

$$
y_{i}=x_{i}^{\prime} \beta+u_{q i}
$$

onde:

$y_{i}$ é o vetor da variável dependente;

$x_{i}^{\prime}$ é o vetor de variáveis explanatórias;

$\beta$ é o vetor de coeficientes;

$\mu_{q i}$ é o resíduo para o q-ésimo quantil.

O valor mínimo dos desvios absolutos $\left(y_{i}-x_{i}^{\prime} \beta\right)$ é dado pela solução do problema:

$$
\min _{\beta} \sum_{t=1}^{n}\left|y_{t}-x_{t}^{\prime} \beta\right|
$$

Esta fórmula pode ser generalizada para que o coeficiente $\beta$ se ajuste a qualquer

\footnotetext{
${ }^{2}$ MQO daqui em diante.
}

${ }^{3}$ LAD doravante. 
outro quantil da distribuição da variável dependente, tem-se:

$$
\begin{aligned}
& \min _{\beta} 1 / n\left\{\sum_{i \varepsilon\left\{i: y_{i} \geq x_{i}^{I} \beta\right\}} q\left|y_{i}-x_{i}^{I} \beta\right|+\sum_{i \varepsilon\left\{i: y_{i} \geq x_{i}^{I} \beta\right\}}(1-q)\left|y_{i}-x_{i}^{I} \beta\right|\right\}= \\
& \min _{\beta} 1 / n \sum \rho_{q}\left(y_{i}-x_{i}^{I} \beta\right)=\min _{\beta} 1 / n \sum \rho_{q}\left(u_{q i}\right)
\end{aligned}
$$

onde a função check, $\rho_{q}\left(\mu_{q i}\right)$, é definida como:

$$
\left\{\begin{array}{c}
\rho_{q}\left(\mu_{q i}\right)=q \mu_{q i} ; \text { se } \mu_{q i} \geq 0 \\
\rho_{q}\left(\mu_{q i}\right)=(q-1) \mu_{q i} ; \text { se } \mu_{q i}<0
\end{array} \quad q \in[0,1]\right.
$$

A variável dependente $y$ é ordenada de forma crescente, ou seja, quanto mais elevado for o quantil - quanto maior for $q$ - mais alto é o valor da variável y dos indivíduos presentes nesse quantil.

O modelo LAD generalizado tem por objetivo minimizar, dada a variável dependente $y$ e suas variáveis explanatórias $x$, a soma dos desvios absolutos para cada quantil da variável $y$. Desse modo, tem-se que a interpretação dos coeficientes é análoga a do método de MQO.

Enquanto este último nos fornece, como resultado, coeficientes que medem a influência das variáveis explanatórias sobre a média condicional da variável dependente, no método de regressões quantílicas, os coeficientes representam a influência das variáveis explanatórias sobre a variável dependente condicional ao q-ésimo quantil. Em outras palavras, a análise através de regressões quantílicas permite analisar toda a distribuição enquanto o método de MQO só permite analisar a média da relação entre as variáveis.

A função check, nada mais é do que a solução para um problema que minimiza a soma ponderada dos desvios absolutos. Desse modo, para quantis acima da mediana, digamos $\theta=0,75$, um maior peso é colocado sobre resíduos acima da mediana que em resíduos abaixo da mediana. Estes ajustes empurram a minimização acima da mediana, e impedem os desvios acima da mediana a se minimizarem mais que os desvios abaixo dela, tornando a estimação mais aderente ao quantil 0,75 .

O estimador LAD generalizado se mostra mais adequado nos caso em que o pesquisador está interessado em analisar partes específicas da distribuição condicional da variável dependente. 
No presente estudo, a estimação do modelo foi realizada utilizando o software estatístico Stata 10.0 para os quantis 0,25;0,50;0,75 e 0,90. Desse modo, têm-se resultados para quatro diferentes níveis de renda dos municípios. A regressão do quantil 0,25 nos fornece resultados aplicados aos municípios com os $25 \%$ menores rendimentos da amostra; já a regressão do quantil 0,90 nos fornece resultados para os municípios com os $90 \%$ maiores rendimentos da amostra. Análoga é a interpretação dos resultados para as regressões dos quantis 0,50 e 0,75 .

\subsection{A BASE DE DADOS}

Neste modelo serão utilizadas proxies de estoques, consumo de energia não residencial por trabalhador para a variável de capital físico, e anos de escola para a variável de capital humano. Todas as variáveis foram divididas pelo número de trabalhadores, que é a população com mais de 15 anos de idade.

O PIB dos municípios foi obtido junto à base de dados do IPARDES e IBGE, e se encontra em preços constantes de 2000. Os dados de consumo de energia não residencial, em MWh, também foram obtidos junto ao IPARDES, sendo o consumo de energia não residencial a diferença entre o consumo total de energia e consumo residencial em cada município. Esta é a variável utilizada como proxy para o estoque de capital físico, por faltarem dados sobre investimentos nos municípios estudados.

Os dados pra anos de estudo foram obtidos junto ao IPEA. Esta variável é utilizada como proxy para capital humano. Os dados para a população com mais de 15 anos são provenientes do IBGE e IPEA, e todas as demais variáveis que estão em nível por trabalhador foram obtidas com base nesta variável.

Quando trabalhamos com dados no estado estacionário, a base de dados é composta por três períodos: 1980, 1991 e 2000. No caso do estado de convergência, utilizamos os períodos de 1991 e 2000, e a diferença do PIB por trabalhador é composta por $\ln (1991)$ $\ln (1980)$ e $\ln (2000)$ - $\ln (1980)$. Adicionalmente, o PIB por trabalhador inicial é para o ano de 1980.

A base de dados é composta por 290 municípios em 3 períodos de análise. Como o número de municípios em 1980 era menor do que nos outros anos (1991 e 2000), os municípios que foram desmembrados a partir de 1980, foram somados aos municípios de origem. As variáveis que já estavam em média, como a variável anos de estudo, foram 
encontradas a partir de uma média ponderada do município de origem com os municípios que foram desmembrados.

\section{RESULTADOS}

Na Tabela 1, podemos ver os resultados para o ano de 1980. Em toda a análise para este período é feita a suposição de que os municípios se encontram no estado estacionário ${ }^{4}$. Nas quatro primeiras colunas, os resultados foram obtidos através do método LAD para os quantis 0,$25 ; 0,50 ; 0,75$ e 0,99 . Na coluna seguinte foi utilizado o método de MQO.

A variável de capital humano apresenta o seu maior valor no quantil 0,25 , onde $1 \%$ de aumento nesta variável reflete um incremento de $0,463 \%$ na renda do município. Como a média de anos de estudo neste período é de 2,38 anos, um ano a mais de estudo leva a um aumento de $42,07 \%$ na quantidade de capital humano. Considerando que a elasticidade da renda é de 0,463 no quantil 0,25, isso implica que para os municípios que estão entre os $25 \%$ com menor renda, um ano a mais de estudo resulta em um aumento na renda de 19,47\%. Somente no quantil 0,99, o coeficiente desta variável apresenta um sinal negativo.

TABELA 1 - RESULTADOS DAS REGRESSÕES PARA 1980

\begin{tabular}{l|r|r|r|r|r}
\hline \multicolumn{1}{c}{ Quantil } & \multicolumn{1}{c}{$\mathbf{0 , 2 5}$} & \multicolumn{1}{c}{$\mathbf{0 , 5 0}$} & \multicolumn{1}{c}{$\mathbf{0 , 7 5}$} & \multicolumn{1}{c}{$\mathbf{0 , 9 9}$} & \multicolumn{1}{c}{ MQO } \\
\hline $\mathbf{H}$ & 0,463 & 0,308 & 0,222 & $-0,559$ & 0.287 \\
& $(0,083)^{* *}$ & $(0.078)^{* *}$ & $(0,089)^{* *}$ & $(0,053)^{* *}$ & $(0,084)^{* *}$ \\
$\mathbf{K}$ & 0,201 & 0,290 & 0,304 & 0,771 & 0,281 \\
& $(0.026)^{* *}$ & $(0.025)^{* *}$ & $(0,028)^{* *}$ & $(0,119)^{* *}$ & $0.027)^{* *}$ \\
$\mathbf{C}$ & 1,497 & 1,901 & 2,142 & 4,404 & 1,910 \\
& $(0.093)^{* *}$ & $(0.088)^{* *}$ & $(0.099)^{* *}$ & $(0,129)^{* *}$ & $(0.094)^{* *}$ \\
$\mathbf{N}$ & 290 & 290 & 290 & 290 & 290 \\
$\mathbf{R}^{\mathbf{2}}$ & & & & & 0,475 \\
\hline
\end{tabular}

NOTA: erro padrão está entre parêntese. ${ }^{*}$ significativo ao nível de $5 \%$. ** significativo ao nível de $1 \%$. A variável dependente é renda por trabalhador. H é anos de escola, K é o consumo de energia não residencial por trabalhador, $\mathrm{C}$ é a constante, $\mathrm{N}$ é o tamanho da amostra.

Na Tabela 2, podemos ver o resultado da regressão para o ano de 1991. Nas quatro primeiras colunas é feita a suposição de que o município está no estado estacionário. Desse modo, testamos a adequação ao método LAD com os quantis 0,$25 ; 0,50 ; 0,75$ e 0,99 . Na quinta coluna testamos a adequação ao método de MQO, com os municípios no estado estacionário.

\footnotetext{
${ }^{4}$ Para um estudo da forma funcional utilizada no presente estudo ver Mankiw, Romer e Weil (1992).
} 
$\mathrm{Na}$ sétima, oitava, nona e décima coluna, assumimos que os municípios estão no estado de convergência. É utilizado o método LAD com os quantis 0,$25 ; 0,50 ; 0,75$ e 0,99 . Na décima primeira coluna, apresentamos os resultados da estimação por MQO, com os municípios no estado de convergência.

Considerando que os municípios estão no estado estacionário, podemos ver que o capital físico é mais importante para explicar os níveis de renda por trabalhador dos diferentes municípios. Para todos os quantis de renda, os coeficientes são positivos e significativos a 1\%. O capital físico tem um coeficiente maior, para o quantil 0,75 , onde um aumento de $1 \%$ no consumo de energia elétrica não residencial leva a um incremento de $0,532 \%$ no PIB por trabalhador do município. Os coeficientes da variável de capital humano, não são significativos a $5 \%$ para nenhum dos quantis.

TABELA 2 - RESULTADOS DAS REGRESSÕES PARA 1991

\begin{tabular}{|c|c|c|c|c|c|c|c|c|c|c|}
\hline \multirow{2}{*}{ Quantil } & \multirow{2}{*}{0,25} & \multirow{2}{*}{0,50} & \multirow{2}{*}{0,75} & \multirow{2}{*}{0,99} & \multirow{2}{*}{ MQO(1) } & \multicolumn{4}{|c|}{ Convergência } & \multirow{2}{*}{$\begin{array}{c}\text { MQO } \\
\text { (2) }\end{array}$} \\
\hline & & & & & & 0,25 & 0,50 & 0,75 & 0,99 & \\
\hline $\mathrm{H}$ & $\begin{array}{r}0,198 \\
(0,149)\end{array}$ & $\begin{array}{r}0,169 \\
(0,163)\end{array}$ & $\begin{array}{l}-0,116 \\
(0,129)\end{array}$ & $\begin{array}{r}0,015 \\
(0,126)\end{array}$ & $\begin{array}{r}0,115 \\
(0,113)\end{array}$ & $\begin{array}{r}-0,122 \\
(0,140)\end{array}$ & $\begin{array}{c}-0,068 \\
(0,157)\end{array}$ & $\begin{array}{r}-0,200 \\
(0.161)\end{array}$ & $\begin{array}{r}-0,010 \\
(0,068)\end{array}$ & $\begin{array}{l}-0,113 \\
(0,110)\end{array}$ \\
\hline $\mathrm{K}$ & $\begin{array}{r}0,461 \\
(0,050)^{* *}\end{array}$ & $\begin{array}{r}0,487 \\
(0,048)^{* *}\end{array}$ & $\begin{array}{r}0,532 \\
(0,030)^{* *}\end{array}$ & $\begin{array}{r}0,293 \\
(0,011)^{* *}\end{array}$ & $\begin{array}{r}0,457 \\
(0,034)^{* *}\end{array}$ & $\begin{array}{r}0,412 \\
(0.053) \\
* *\end{array}$ & $\begin{array}{r}0,369 \\
(0,049) \\
* *\end{array}$ & $\begin{array}{r}0,419 \\
(0,037) \\
* *\end{array}$ & $\begin{array}{r}0,204 \\
(0,011) \\
* *\end{array}$ & $\begin{array}{r}0,371 \\
(0,034) \\
* *\end{array}$ \\
\hline C & $\begin{array}{r}1,422 \\
(0,199)^{* *}\end{array}$ & $\begin{array}{r}1,707 \\
(0,214)^{* *}\end{array}$ & $\begin{array}{r}2,120 \\
(0,166)^{* *}\end{array}$ & $\begin{array}{r}2,615 \\
(0,145)^{* *}\end{array}$ & $\begin{array}{r}1,743 \\
(0,148)^{* *}\end{array}$ & $\begin{array}{r}1,202 \\
(0,200) \\
* *\end{array}$ & $\begin{array}{r}1,250 \\
(0,211) \\
* *\end{array}$ & $\begin{array}{r}1,636 \\
(0,215) \\
* *\end{array}$ & $\begin{array}{r}2,245 \\
(0,029) \\
* *\end{array}$ & $\begin{array}{r}-0,659 \\
(0,049) \\
* *\end{array}$ \\
\hline Y80 & & & & & & $\begin{array}{r}-0,655 \\
(0,077) \\
* *\end{array}$ & $\begin{array}{r}-0,620 \\
(0,070) \\
* *\end{array}$ & $\begin{array}{r}-0,630 \\
(0,067) \\
* *\end{array}$ & $\begin{array}{r}-0,818 \\
(0,029) \\
* *\end{array}$ & $\begin{array}{r}1,384 \\
(0,147) \\
* *\end{array}$ \\
\hline $\mathrm{N}$ & 290 & 290 & 290 & 290 & 290 & 290 & 290 & 290 & 290 & 290 \\
\hline $\mathrm{R}^{2}$ & 0,224 & 0,296 & 0,322 & 0,403 & 0,377 & 0,231 & 0,229 & 0,220 & 0,274 & 0,442 \\
\hline
\end{tabular}

NOTA: erro padrão está entre parêntese. ${ }^{*}$ significativo ao nível de $5 \%$. ** significativo ao nível de $1 \%$. A variável dependente é renda por trabalhador nas quatro primeiras regressões, nas regressões seguintes a variável dependente é a variação da renda por trabalhador. H é anos de escola, K é o consumo de energia não residencial por trabalhador. C é a constante, Y80 é o nível de renda por trabalhador em 1980 e N é o tamanho da amostra.

Na Tabela 3, podemos ver os resultados das regressões para o ano 2000. Nas quatro primeiras colunas é feita a suposição de que os municípios estão no estado estacionário. As quatro primeiras colunas foram testadas através do Método LAD, com os quantis 0,$25 ; 0,50$; 0,75 e 0,99 . Na quinta coluna testamos a adequação ao método de MQO, com os municípios no estado estacionário. Da sétima coluna em diante, assumimos que os municípios se encontram em estado de convergência. 
Para o ano de 2000, os resultados se mostraram muito mais expressivos, Com os municípios no estado estacionário, a variável de capital humano tem um efeito maior sobre a renda. O maior coeficiente desta variável se dá no quantil 0,50 (mediana), onde o aumento de $1 \%$ na variável, reflete em um aumento de $0,536 \%$ na renda do município. Como a média de anos de escola neste período é de 4,67, um ano a mais de escola representa um aumento de $21,41 \%$ na quantidade de capital humano. Como a elasticidade da renda em relação ao capital humano é de 0,536\%, em média, um ano a mais de estudo para os municípios que estão se quantil reflete em um aumento na renda de $11,47 \%$.

TABELA 3 - RESULTADOS DAS REGRESSÕES PARA 2000

\begin{tabular}{|c|c|c|c|c|c|c|c|c|c|c|}
\hline \multirow{2}{*}{ Quantil } & \multirow{2}{*}{0,25} & \multirow{2}{*}{0,50} & \multirow{2}{*}{0,75} & \multirow{2}{*}{0,99} & \multirow{2}{*}{ MQO(1) } & \multicolumn{4}{|c|}{ Convergência } & \multirow{2}{*}{ MQO(2) } \\
\hline & & & & & & 0,25 & 0,50 & 0,75 & 0,99 & \\
\hline $\mathrm{H}$ & $\begin{array}{r}0,242 \\
(0,105)^{*}\end{array}$ & $\begin{array}{r}0,536 \\
(0,124)^{* *}\end{array}$ & $\begin{array}{r}0,506 \\
(0,148)^{* *}\end{array}$ & $\begin{array}{r}0,411 \\
(0,308)\end{array}$ & $\begin{array}{r}0,453 \\
(0,123)^{* *}\end{array}$ & $\begin{array}{r}0,358 \\
(0,164) \\
*\end{array}$ & $\begin{array}{r}0,365 \\
(0,125) \\
* *\end{array}$ & $\begin{array}{r}0,224 \\
(0,175)\end{array}$ & $\begin{array}{r}-1,700 \\
(0,197) \\
* *\end{array}$ & $\begin{array}{r}0,281 \\
\left(0,128^{*}\right)\end{array}$ \\
\hline $\mathrm{K}$ & $\begin{array}{r}0,300 \\
(0,026)^{* *}\end{array}$ & $\begin{array}{r}0,295 \\
(0,029)^{* *}\end{array}$ & $\begin{array}{r}0,364 \\
(0,031)^{* *}\end{array}$ & $\begin{array}{r}0,252 \\
(0,030)^{* *}\end{array}$ & $\begin{array}{r}0,285 \\
(0,029)^{* *}\end{array}$ & $\begin{array}{r}0,197 \\
(0,038) \\
* *\end{array}$ & $\begin{array}{r}0,252 \\
(0,027) \\
* *\end{array}$ & $\begin{array}{r}0,336 \\
(0,038) \\
* *\end{array}$ & $\begin{array}{r}0,527 \\
(0,024) \\
* *\end{array}$ & $\begin{array}{r}0,252 \\
(0,030)^{* *}\end{array}$ \\
\hline $\mathrm{C}$ & $\begin{array}{r}1,376 \\
(0,162)^{* *}\end{array}$ & $\begin{array}{r}1,065 \\
(0,190)^{* *}\end{array}$ & $\begin{array}{r}1,298 \\
(0,227)^{* *}\end{array}$ & $\begin{array}{r}2,230 \\
(0,534)^{* *}\end{array}$ & $\begin{array}{r}1,232 \\
(0,188)^{* *}\end{array}$ & $\begin{array}{r}0,893 \\
(0,233) \\
* *\end{array}$ & $\begin{array}{r}0,969 \\
(0,180) \\
* *\end{array}$ & $\begin{array}{r}1,363 \\
(0,263) \\
* *\end{array}$ & $\begin{array}{r}4,031 \\
(0,274) \\
* *\end{array}$ & $\begin{array}{r}1,192 \\
(0,184)^{* *}\end{array}$ \\
\hline Y80 & & & & & & $\begin{array}{r}-0,830 \\
(0,067) \\
* *\end{array}$ & $\begin{array}{r}-0,804 \\
(0,043) \\
* *\end{array}$ & $\begin{array}{r}-0,800 \\
(0,058) \\
* *\end{array}$ & $\begin{array}{r}-0,286 \\
(0,046) \\
* *\end{array}$ & $\begin{array}{r}-0,832 \\
(0,044)^{* *}\end{array}$ \\
\hline $\mathrm{N}$ & 290 & 290 & 290 & 290 & 290 & 290 & 290 & 290 & 290 & 290 \\
\hline $\mathrm{R}^{2}$ & 0,230 & 0,246 & 0,237 & 0,137 & 0,377 & 0,396 & 0,336 & 0,259 & 0,218 & 0,570 \\
\hline
\end{tabular}

NOTA: erro padrão está entre parênteses. ${ }^{*}$ significativo ao nível de $5 \%$. ${ }^{* *}$ significativo ao nível de $1 \%$. A variável dependente é a renda por trabalhador nas quatro primeiras regressões, nas regressões seguintes a variável dependente é a variação da renda por trabalhador. H é anos de escola, $\mathrm{K}$ é o consumo de energia não residencial por trabalhador. C é a constante, Y80 é o nível de renda por trabalhador em 1980 e N é o tamanho da amostra.

\section{CONCLUSÃO}

A principal conclusão do presente estudo é que ocorreu uma grande variação na importância do capital humano em cada quantil de 1980 até 2000. Enquanto o capital humano era mais importante para os níveis de renda mais baixos em 1980, este passou a ser mais relevante para os níveis mais elevados de renda em 2000, principalmente para o quantil 0,50 .

Desse modo, os resultados apresentados indicam que esse fator tem se tornado mais relevante para as classes de renda mais elevadas. Ou seja, a educação formal tem apresentado maior interação com cargos de renda mais elevada que, em média, são mais complexos. 


\section{REFERÊNCIAS}

KOENKER, R. HALLOCK, K. F. Quantile Regression. Journal of Economic Perspectives, v. 15, n. 4, p. 143-156, 2001.

KOENKER, R.; BASSET, G. Regression Quantiles. Econométrica, n. 46, 1978.

MANKIW, N.G. ROMER, D. WEIL, D.N. (1992), A Contribution to the empirics of Economic Growth, The Quarterly Journal of Economics, vol. 107, no. 2, pp. 407-37, 1992.

SOLOW, R. M. A contribution to the theory of economic growth. The Quarterly Journal of Economics, v.70, n.1, p.65-94, 1956. 